\title{
Imaging Spectrum of Unusual Pineal Region Masses: A Short Series of Four Cases
}

\author{
Anandamoyee Dhar ${ }^{1}$ Ruchi Jain ${ }^{1}$ Bipin Walia ${ }^{2} \quad$ Urmi Mukherjee $^{3} \quad$ Bharat Aggarwal $^{1}$ \\ ${ }^{1}$ Department of Radio-Diagnosis, Max Super Specialty Hospital, \\ New Delhi, India \\ 2 Department of Neurosurgery, Max Super Specialty Hospital, \\ New Delhi, India \\ ${ }^{3}$ Department of Pathology, Max Super Specialty Hospital, \\ New Delhi, India \\ Address for correspondence Anandamoyee Dhar, MBBS, MD, \\ Department of Radio-Diagnosis, Max Super Specialty Hospital, \\ 1 Press Enclave Road, New Delhi 110017, India \\ (e-mail: anandamoyee@gmail.com). \\ Indian J Neurosurg 2017;6:135-140.
}

\begin{abstract}
Pineal tumors are rare intracranial neoplasm with germ cell tumors and pineal parenchymal tumors being the predominant type, seen in 80 to $90 \%$ of cases. Only a

Keywords

- pineal region tumor

- glioblastoma of pineal region

- pineal astrocytoma few cases of glioblastomas have been reported. The incidence of astrocytoma and meningioma is also low in this region. In this article, we will discuss the key imaging features and diagnostic dilemmas in four rare types of pineal region tumors including pineal parenchymal tumor of intermediate differentiation, pineal astrocytoma, falcotentorial junction meningioma, and glioblastoma with subependymal spread.
\end{abstract}

\section{Introduction}

Pineal tumors are rare intracranial neoplasm accounting for 0.5 to $1 \%$ of all intracranial neoplasm in adults and 1 to $2.7 \%$ in children. ${ }^{1}$ The most common tumors encountered in this region are germ cell tumors accounting for $\sim 50$ to $75 \%$ cases $^{2}$ and pineal parenchymal tumors accounting for 14 to $27 \%$ of tumors. $^{3}$ Other rare tumors are astrocytomas, tumors arising from adjacent meninges such as meningioma, and tumor-like lesions such as epidermoid, dermoid cyst, or lipoma. The imaging characteristics are nonspecific and overlapping. The knowledge of the clinical presentation and imaging finding may help in the diagnosis and characterization of these rare entities.

\section{Case Series}

Case 1: A 27-year-old man presented with holocranial dull headache, blurring of vision, occasional vomiting, and imbalance for a short duration. Magnetic resonance imaging (MRI) of the brain revealed a well defined lobulated mass in the pineal region. The mass was splaying and indenting on the posterior part of third ventricle, dorsal midbrain, and the splenium of corpus callosum (see - Fig. 1a, b). The lesion appeared hyperintense on $\mathrm{T} 2 \mathrm{~W}$ images and isointense to gray matter on T1W images with intense but heterogeneous contrast enhancement with few cystic areas within the lesion (see - Fig. 1c). Marked hydrocephalus was present involving lateral and third ventricles. No fat-containing areas were seen. The central part of the lesion showed blooming on gradient images suggestive of engulfment of calcification. Restricted diffusion with decreased apparent diffusion coefficient (ADC) values was present (see - Fig. 1d, e). Pineal gland was not separately visualized. Imaging diagnosis of germinoma was considered in view of mass in the pineal region showing intense enhancement with only few cystic areas, central engulfed calcification, no definite invasion of adjacent structures, and diffusion restriction. Biomarkers were, however, not assessed preoperatively and immediate surgery was performed. Occipital burr hole and Ommaya reservoir placement were done with midline suboccipital craniotomy with supracerebellar approach and gross total tumor resection. Frozen section revealed round cell with moderate-to-high cellularity. Microscopy showed sheets of polygonal cells with thin incomplete fibrous septae, mild-to-moderate nuclear atypia, and few mitotic figures. Immunohistochemistry revealed synaptophysin expression on tumor cells and Glial received

April 8, 2015

accepted

September 17, 2016

published online

February 10, 2017
DOI https://doi.org/

10.1055/s-0036-1596044. ISSN 2277-954X. (c) 2017 Neurological Surgeons' Society
of India

License terms

(๑) $\Theta \circledast$ 

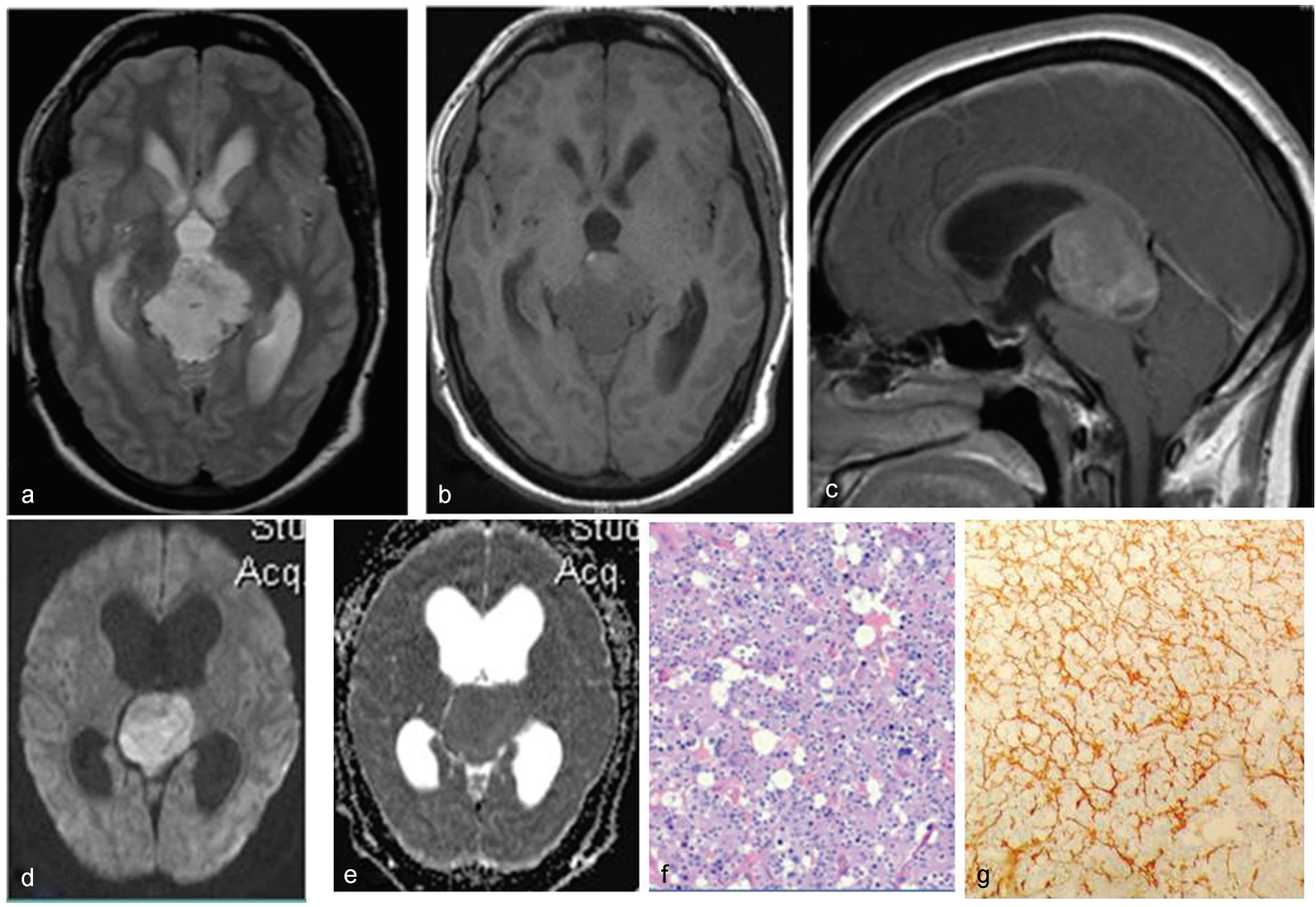

Fig. 1 (a, b) Axial T2W images show a well-defined lobulated mass lesion in the pineal region appearing hyperintense on T2W images and isointense to gray matter on T1W images. (c) Postcontrast axial images reveal intense heterogeneous enhancement. (d, e) DWI and ADC images reveal mild restricted diffusion with decreased apparent diffusion. (f) It shows round-to-polygonal cells arranged in sheets with interspersed incomplete fibrous septae $(\mathrm{H}$ and $\mathrm{E} \times 200)$. $(\mathrm{g})$ It shows expression of synaptophysin on tumor cells $(\mathrm{GFAP} \times 100)$.

fibrillary acidic protein (GFAP) expression on peripheral astrocytes (see - Fig. 1f, g), suggesting a pineal parenchymal tumor of intermediate differentiation (PPTID).

Case 2: A 52-year-old woman presented with headache, progressively decreasing vision, and frequent episodes of falls over the past 1 year. On examination, Romberg's test was positive. Ophthalmological examination revealed decreased visual acuity, bilateral temporal field defects, pale disc with blurred margins, and bilateral papilledema with partial optic disc atrophy likely due to longstanding increased intracranial pressure. MRI of brain revealed a large extra-axial lesion appearing isointense to gray matter on T1W images and hyperintense on T2W images (see - Fig. 2a, b). Postcontrast images showed homogeneous enhancement and a broad attachment to the posterior falx and tentorium with compression of the aqueduct of sylvius and proximal ventricular dilatation (see - Fig. 2 c). There was no evidence of restricted diffusion or blooming on gradient images. (see - Fig. 2d, e). Noncontrast CT (NCCT) of the brain revealed a well defined hyperdense lesion (see - Fig. 2f). There was compression of optic radiation posterolaterally by the tumor, which probably explained the field defects. The diagnosis of meningioma was considered, and tumor excision was performed with right Poppen's approach after tumor embolization to decrease vascularity. Microscopic examination revealed interlacing fascicles of spindle cells with uniform nuclei and indistinct cytoplasm on the background of collagen rich matrix with frequent psammoma bodies. No nuclear atypia was seen, thus confirming the diagnosis of fibroblastic meningioma WHO grade 1 (see - Fig. $\mathbf{2 g}$ ).

Case 3: A 31-year-old woman presented to the neurology OPD with severe headache and vomiting for 1 year. The neurological examination was normal except for mild papilledema on ophthalmoscopy. The brain MRI revealed a lobulated lesion appearing iso- to hyperintense on $\mathrm{T} 2 \mathrm{~W}$ and mildly hyperintense to gray matter on T1W images (see - Fig. 3a, b). No significant contrast enhancement was seen on postgadolinium images (see - Fig. 3c). The tumor revealed mild diffusion restriction with low ADC values. However, no evidence of blooming was seen on the gradient images to suggest calcification (see - Fig. 3d) (see - Fig. 3e, f). Suboccipital craniotomy with supracerebellar approach and microscopic tumor resection was performed after initial decompression by ventriculoperioteal shunt placement. During surgery, the tumor was very vascular contrary to the observation of low-contrast enhancement on MRI. Histopathogy showed round-to-oval mildly pleomorphic nuclei with fibrillary background (see - Fig. $\mathbf{3 g}$ ). There were no mitotic figures or necrosis or microvascular proliferation. GFAP positivity was seen strongly on immunohistochemistry 

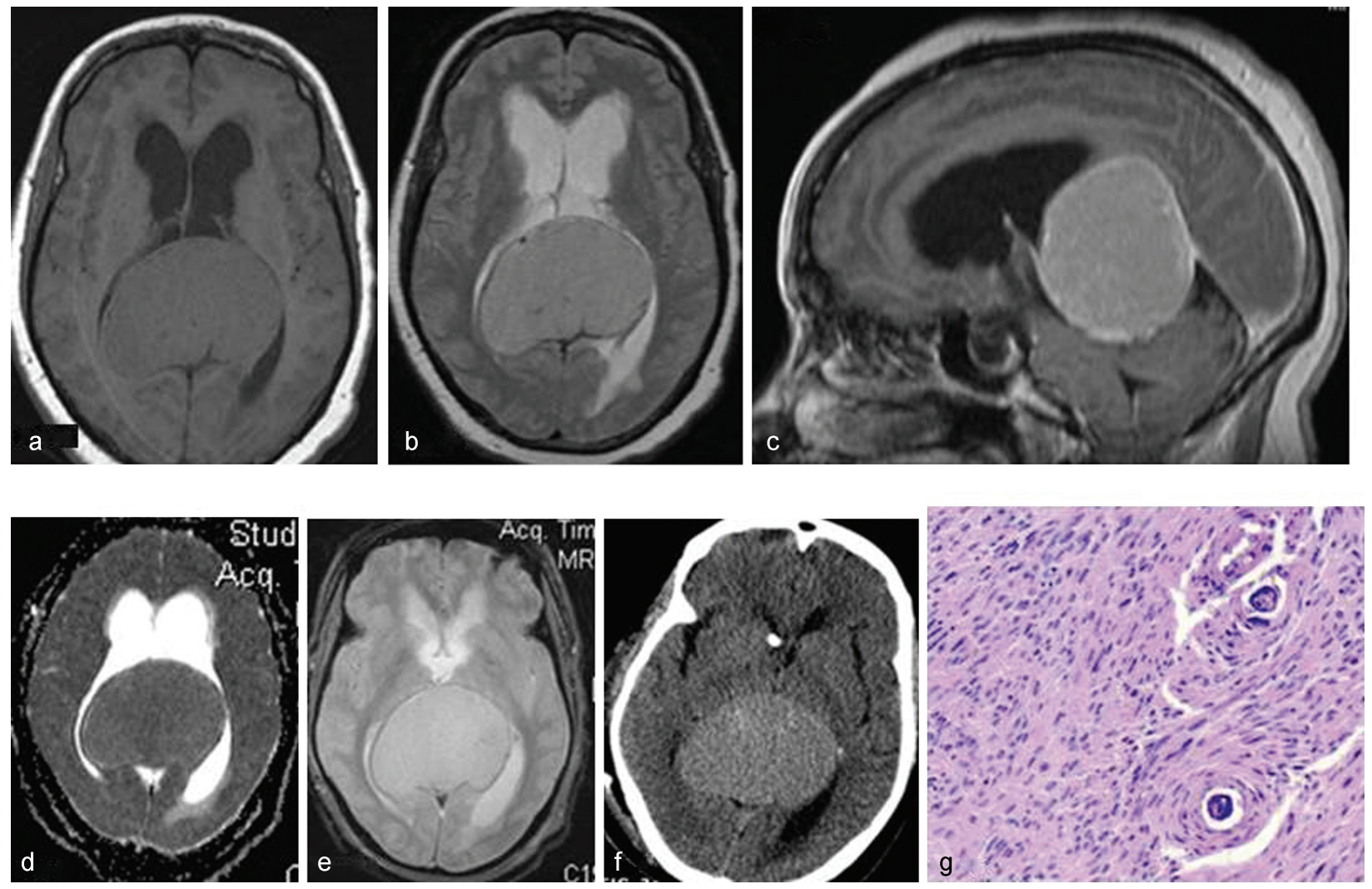

Fig. 2 (a, b) Axial T1 and T2 images show a T1W isointense and T2W hyperintense extra-axial lesion in pineal region with obstructive hydrocephalus. (c) Postcontrast sagittal images reveal homogeneous enhancement with broad attachment to falx. No restricted diffusion seen with no hypointensity on ADC maps and no blooming on GRE images (d, e). (f) NCCT brain shows a well-defined hyperdense lesion in the pineal region. $(\mathbf{g})$ It shows interlacing fascicle of ovoid to spindle cells with uniform nuclei and indistinct cytoplasmic margin on rich background of collagen matrix and psammoma bodies $(H \& E \times 200)$.

with absent synaptophysin and Neuron specefic enolase (NSE) expression; KI 67 proliferation index was 6 to $8 \%$ (see - Fig. 3h), which indicated the diagnosis of astrocytoma WHO grade II.

Case 4: A 37-year-old male patient presented with progressive quadriparesis over past few months with drooping eyelids. Examination revealed third nerve palsy with impaired ocular convergence. A heterogeneously enhancing mass was detected on brain MRI in the pineal region. The mass was infiltrating into the tectum and midbrain (see - Fig. 4a, b). Linear enhancement was seen along the third ventricle suggestive of subependymal spread (see - Fig. 4c); however, no other satellite lesion was seen in rest of the craniospinal axis. This lesion showed central blooming on GRE images suggestive of calcification (see -Fig. 4d). An anterior cystic component with mild diffusion restriction was also seen (see - Fig. 4e, f). There was compression on the aqueduct of sylvius with obstructive hydrocephalus, periventricular ooze, and effacement of basal cistern. Tumor decompression was done via supracerebellar infratentorial approach. Histopathology revealed cellular astrocytic growth with marked nuclear pleomorphism, foci of necrosis, and proliferation with strong expression of GFAP confirming the diagnosis of glioblastoma multiformes grade IV (see - Fig. 4g, h).

\section{Discussion}

Pineal gland is a small structure measuring 10 to $14 \mathrm{~mm}$ in size $^{4}$ and is located along midline above the superior colliculus in relation to the posterior border of the third ventricle. It plays a role in regulating sleep and wake cycle, seasonal functions, and secretion of melatonin. Normal gland comprises pineocyte (95\%) and astrocytes (5\%), and calcification is commonly seen in the gland.

The common clinical presentation of pineal tumors is due to mass effect, manifesting as raised intracranial pressure and obstructive hydrocephalus, which may present as headache, blurring of vision, and vomiting. A rare presentation is precocious puberty. Parinaud's syndrome may occur, due to compression and infiltration of the quadrigeminal plate, and constitutes difficulty of upward vertical gaze, mydriasis, blepharospasm, and impaired ocular convergence. ${ }^{5,6}$

On imaging, pineal parenchymal lesions are usually well circumscribed and solid. They are seen to arise from pineocytes and expand the pineal gland to produce the characteristic "explosion" of normal pineal calcifications toward the periphery. ${ }^{7}$ Infiltration into the adjacent structures, hemorrhage, and necrotic changes are seen in pineaoblastoma due to its high malignant nature. ${ }^{7}$ 

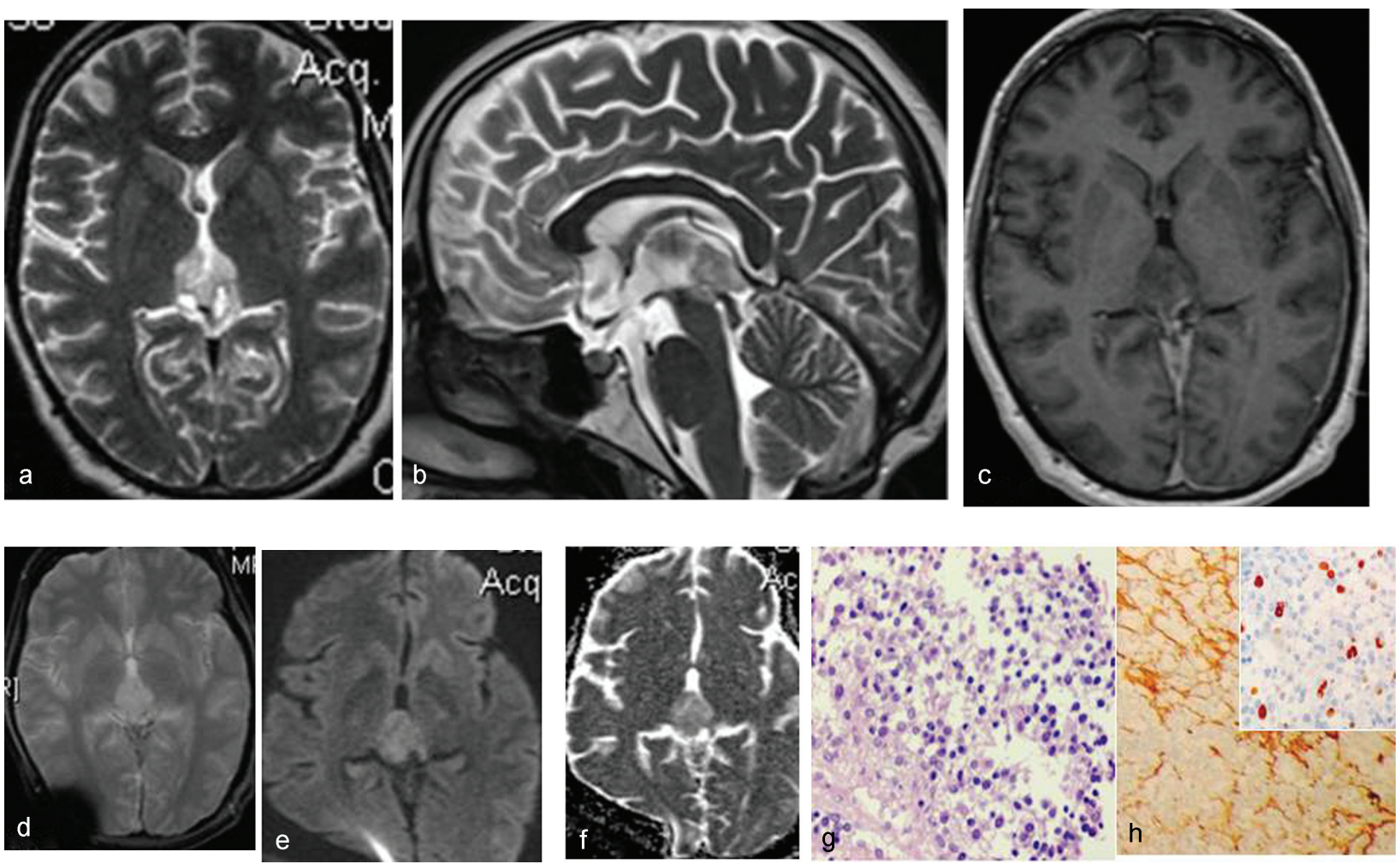

Fig. 3 (a, b) T2W axial and sagittal images show a lobulated mildly hyperintense lesion in the pineal region superior to the colliculus. (c) Axial postcontrast: No significant enhancement seen on postgadolinium images. (d) GRE images show no calcification within the lesion. (e, f) These show mild restricted diffusion and low ADC values in this lesion. $(g)$ It shows round-to-oval mildly pleomorphic tumor cells with uniform chromatin in a fibrillary background (H\&E $\times 200$ ). (h) It shows strong GFAP expression with 6 to $8 \%$ KI67 proliferation index (inset, GFAP $\times 100$ ).
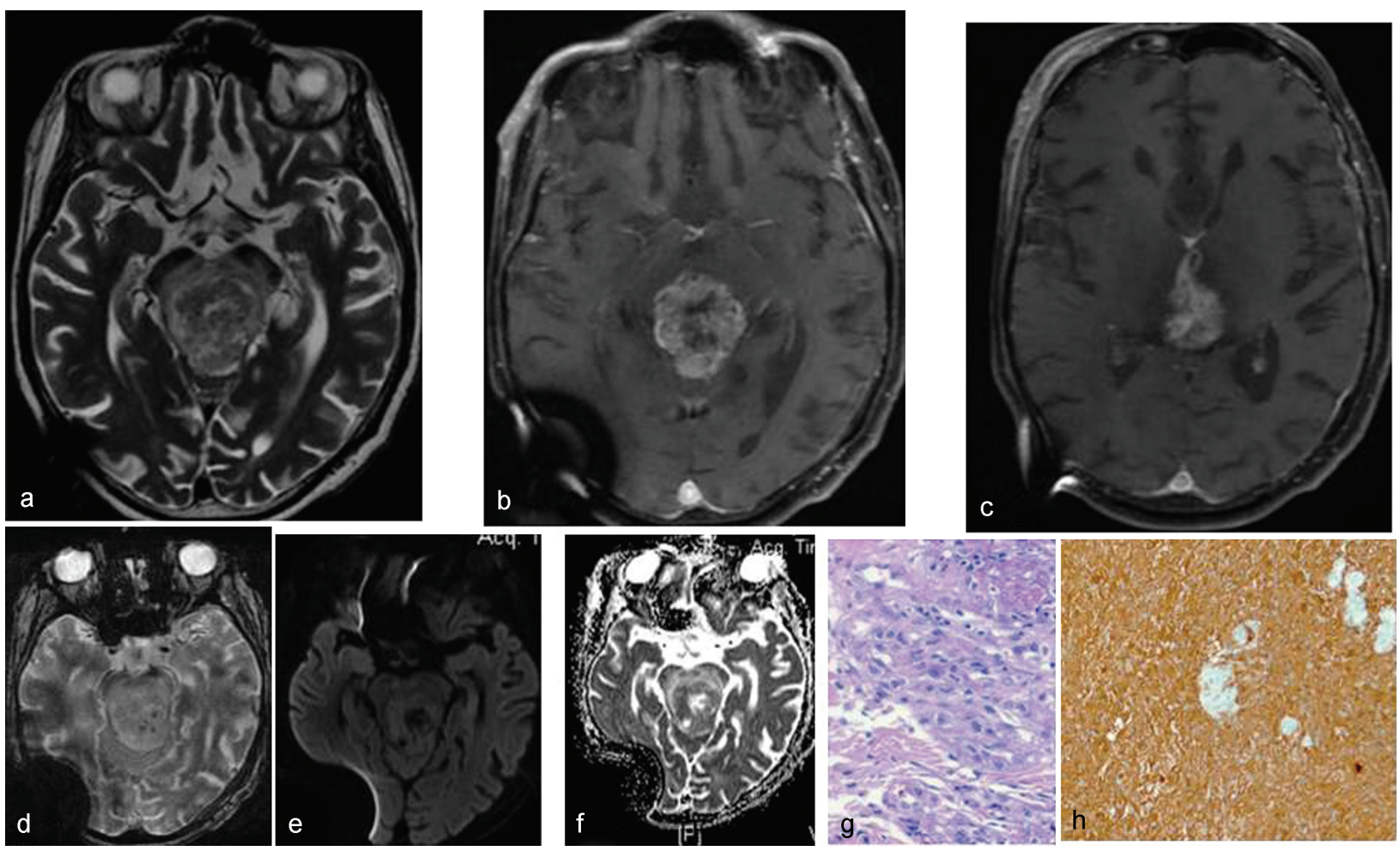

Fig. 4 (a) A hetrogeneously T2W hyperintense lesion in pineal region with infiltration in tectum and midbrain and obstructive hydrocephalous. (b) Postcontrast axial images show heterogeneous enhancement with areas of necrosis. (c) Linear enhancement is seen along the third ventricle. (d) Axial GRE images show central blooming suggestive of calcification. (e, f) DWI and ADC images show mild restricted diffusion. (g) It shows cellular astrocytic growth with marked cellular pleomorphism and necrosis $(H \& E \times 200)$. (h) It shows strong expression of GFAP $(G F A P \times 100)$. 
Germ cell tumors are classified as germinomas and nongerminomatous tumors. ${ }^{8}$ They appear as solid avidly enhancing tumors with subependymal or leptomeningeal or intraventricular spread. Cystic component is present in 20 to $25 \%$ cases, $^{7}$ and the solid component may demonstrate low ADC values. ${ }^{9,10}$ Nongerminomatous germ cell tumors like teratomas show higher heterogeneity with multicystic appearance, fat, and calcification. ${ }^{7}$ Choriocarcinomas, yolk sac tumor, and embryonal cell tumors constitute $\sim 10 \%$ of nongerminomatous germ cell tumors and are more heterogeneous due to hemorrhage and cystic changes and often have high serum or Cerebrospinal fluid (CSF) oncoproteins. ${ }^{11}$ In our series, heterogeneity and diffusion restriction were seen in the case of PPTID and glioblastoma, misleading the diagnosis.

Pineal region meningiomas usually arise from tela choroidea, falcotentorial junction, or velum interpositum. ${ }^{6}$ They are intensely enhancing dural-based lesions, which show low-to-intermediate signal on T1W images and intermediate-to-high signal on T2W images. Diffusion restriction is an important diagnostic feature and seen in solid component of densely packed tumors, like pineoblastomas, germinoma, and meningioma. In our case series, diffusion restriction was seen in PPTID and glioblastoma in contrast to meningioma, which revealed no diffusion restriction. Broad dural-based lesion, attachment to dura with a characteristic dural tail, guided the diagnosis of meningioma, which otherwise would have been difficult as the pineal gland was compressed due to large size of the tumor.

The tissue differentiation of various types of pineal tumors is difficult on imaging and is based on detection of type of calcification, solid appearance with diffusion restriction, heterogeneity, cystic changes, and subependymal spread. Peripheral dispersion of calcification is a feature of pineoblastoma in contrast to central engulfment of calcification in germinomas. - Table $\mathbf{1}$ summarizes the imaging characteristics of all four cases with their provisional imaging diagnosis and the final histopathological diagnosis. Central engulfment of calcification was seen in the case of PPTID and glioblastoma in our series. Pineal tumors such as pineoblastomas and germinomas usually show marked contrast enhancement. In contrast to other tumors, astrocytoma revealed heterogeneity with insignificant contrast enhancement in our series. Therefore, poor contrast enhancement and heterogeneity may suggest a rarer glial tumor.

Few characteristic features usually observed in germinomas are seen in the case of glioblastoma in our series, like diffuse enhancement, cystic change and subependymal spread along third ventricle. Glioblastomas are extremely rare tumors in this region and only 18 cases have been reported in literature. ${ }^{12}$ Moon et al reviewed the literature ${ }^{12}$ and have also described subependymal and leptomeningeal spread in cases of glioblastomas, as seen in our case.

It may not be possible always to categorize pineal region tumors in a specific subgroup based on imaging pattern. The role of imaging is to primarily provide information about the size of the lesion, extent of specially superior and lateral extent, vascularity and nature of its contents (homogeneity or heterogeneity with fat, cystic, or hemorrhagic areas), the tumor margins, and probability of invasion into adjacent structure. The anatomical relationship of the tumor and surrounding structure like third ventricle, corpus callosum, and compression on quadrigeminal plate, aqueduct, cerebellar vermis, and deep venous system are important for deciding the surgical strategy.

The use of cerebrospinal fluid (CSF) and serum laboratory studies may further help in narrowing the differential diagnosis. Imaging of the entire spine to detect any drop metastases is

Table 1 Summary of imaging appearance of the pineal tumors in present series, their imaging diagnosis, and final histopathological diagnosis

\begin{tabular}{|l|l|l|l|l|}
\hline & Case I & Case II & Case III & Case IV \\
\hline T1W & Hypointense & Intermediate & Hypointense & Hypointense \\
\hline T2W & Hyperintense & Iso to mildly hyperintense & Iso-hyperintense & Hyperintense \\
\hline Margin & Irregular & Well defined & Irregular & III defined \\
\hline Heterogeneity & ++ & - & + & +++ \\
\hline Calcification & Peripheral & - & Central & Central \\
\hline Enhancement & Homogeneous & Homogeneous & Absent & Heterogeneous \\
\hline Restricted diffusion & ++ & - & + & + \\
\hline Cystic changes & + & - & + & - \\
\hline Fat & - & - & - & - \\
\hline Subependymal spread & - & - & - & + \\
\hline Dural enhancement & - & + & Pineal tumor-indeterminate & Germinoma \\
\hline Imaging diagnosis & Germinoma & Meningioma & Astrocytoma & Glioblastoma \\
\hline Histopathology & PPTID & Meningioma & & - \\
\hline
\end{tabular}


important before an aggressive curative surgery is planned.

The first line of treatment for pineal region tumors is surgery; however, palliative shunt may be placed for hydrocephalus prior to definitive surgery. Pineal tumors are deep seated, and different approaches and strategy are used for surgery. ${ }^{13,14}$ In patients with pineal tumors with noncommunicating hydrocephalus, endoscopic third ventriculostomy (ETV) and tumor biopsy are an emerging therapeutic and diagnostic procedures. ${ }^{15}$ Combining endoscopic biopsy with ETV helps in treating hydrocephalus and to obtain CSF for tumor markers and cytology. Moreover, direct evaluation of the surrounding structures identifies malignant dissemination. The current surgical approaches are infratentorial supracerebellar, occipital transtentorial, and transcallosal interhemispherical approach. $^{13,14}$ The infratentorial and supracerebellar approach is the most common used surgical approach to reach the center of the pineal tumor. Moreover, this approach is anterior to velum interpositum and deep venous system to which tumor may be adherent, thus reducing the risk of vascular damage. If the tumor extends superiorly, involving or destroying the corpus callosum or deflecting the venous system or extending lateral to the region of trigone, occipital transtentorial or transcallosal interhemispherical approach is used. Midline suboccipital craniotomy with supracerebellar approach was used in our cases, and presurgery tumor embolization was done in the case of meningioma. The extent of the surgical removal and the histopathogical diagnosis are the main factors deciding the outcome. ${ }^{13}$ Chemotheraphy and sterotactic radiosurgery are the newer effective adjuvant treatment modalities used in malignant tumors.

\section{Conclusion}

Pineal region tumors are rare and primarily constitute germ cell and tumors of pineal parenchymal origin. A wide variety of uncommon tumors like gliomas, meningiomas, and glioblastomas are occasionally encountered. Imaging plays an important role in diagnosis, staging of the disease and excluding satellite lesion and drop metastasis in spine, and helping in deciding the surgical approach. The imaging findings are overlapping; they may be indicative but are often nonspecific. The MRI features such as pattern of calcification, heterogeneity, diffusion restriction, and contrast enhancement help in differentiating these tumors. Leptomeningeal and subependymal spread in glioblastoma and poor contrast enhancement in astrocytomas are the unique MRI features highlighted in our case series.

\section{References}

1 Statistical report: primary brain tumors in the United States, 1998-2002. Hinsdale (Illinois): Central Brain Tumor Registry of the United States; 2005

2 Kersh CR, Constable WC, Eisert DR, et al. Primary central nervous system germ cell tumors. Effect of histologic confirmation on radiotherapy. Cancer 1988;61(11):2148-2152

3 Hirato J, Nakazato Y. Pathology of pineal region tumors. J Neurooncol 2001;54(3):239-249

4 Sumida M, Barkovich AJ, Newton TH. Development of the pineal gland: measurement with MR. AJNR Am J Neuroradiol 1996; 17(2):233-236

5 Klein P, Rubinstein LJ. Benign symptomatic glial cysts of the pineal gland: a report of seven cases and review of the literature. J Neurol Neurosurg Psychiatry 1989;52(8):991-995

6 Konovalov AN, Spallone A, Pitzkhelauri DI. Meningioma of the pineal region: a surgical series of 10 cases. J Neurosurg 1996; 85(4):586-590

7 Smith AB, Rushing EJ, Smirniotopoulos JG. From the archives of the AFIP: lesions of the pineal region: radiologic-pathologic correlation. Radiographics 2010;30(7):2001-2020

8 Louis DN, Ohgaki H, Wiestler OD, Cavenee WK. WHO Classification of Tumours of the Central Nervous System. Lyon, France: International Agency for Research on Cancer; 2007

9 Douglas-Akinwande AC, Ying J, Momin Z, Mourad A, Hattab EM. Diffusion-weighted imaging characteristics of primary central nervous system germinoma with histopathologic correlation: a retrospective study. Acad Radiol 2009;16(11): 1356-1365

10 Moon WK, Chang KH, Han MH, Kim IO. Intracranial germinomas: correlation of imaging findings with tumor response to radiation therapy. AJR Am J Roentgenol 1999;172(3):713-716

11 Horowitz MB, Hall WA. Central nervous system germinomas. A review. Arch Neurol 1991;48(6):652-657

12 Moon KS, Jung S, Jung TY, Kim IY, Lee MC, Lee KH. Primary glioblastoma in the pineal region: a case report and review of the literature. J Med Case Reports 2008;2:288

13 Konovalov AN, Pitskhelauri DI. Principles of treatment of the pineal region tumors. Surg Neurol 2003;59(4):250-268

14 Konovalov AN, Pitskhelauri DI. Principles of treatment of the pineal region tumors. Surg Neurol 2003;59(4):250-268

15 Morgenstern PF, Osbun N, Schwartz TH, Greenfield JP, Tsiouris AJ, Souweidane MM. Pineal region tumors: an optimal approach for simultaneous endoscopic third ventriculostomy and biopsy. Neurosurg Focus 2011;30(4):E3 